\title{
Comparison of five approaches to keeping power line maintainers' hands warm during work in the cold
}

\author{
Hunt, $\mathrm{S}^{\mathrm{a}}$, and Wells, $\mathrm{R}^{\mathrm{b}, \mathrm{c}^{*}}$ \\ ${ }^{a}$ Toronto Hydro, Toronto, Ontario, Canada \\ ${ }^{\mathrm{b}}$ Department of Kinesiology, University of Waterloo, Waterloo, Ontario, Canada \\ ${ }^{\mathrm{c}}$ Centre of Research Expertise for the Prevention of Musculoskeletal Disorders (CRE-MSD), University of \\ Waterloo, Waterloo, Ontario, Canada
}

\begin{abstract}
Electric utility workers in Canada must frequently work in the cold and must wear thick rubber gloves which can result in rapid fatigue and reduced performance. The purpose of the study was therefore to document the challenge of working in the cold wearing the standard five-finger rubber gloves and covers and compare them to two equipment options, mitten style gloves or a prototype wool liner, and two heating options, glove or torso heating. The dependent measures were grip force, temperature, dexterity (modified Purdue pegboard test and a simulated occupational task), finger sensitivity (Von Frey hair test), perceived effort and thermal sensation. The study population consisted of 10 experienced male utility workers. They worked in a controlled temperature walk-in chamber (-20 degrees Celsius) and performed simulated utility work for 45 minutes with interspersed test batteries. The mitten style glove and woolen liner in a standard glove reduced the effects of working in the cold compared to the standard five-fingered variety with a thin cotton liner. We found that the mitten style glove showed lesser drops in skin temperature for the 3rd and 5th digits than the other conditions $(\mathrm{p}<0.05)$.
\end{abstract}

Keywords: Gloves, strength, performance, temperature, dexterity

\section{Introduction}

Powerline maintainers, also known as electric utility workers, in Canada must frequently work in the cold. They must wear thick rubber gloves to protect themselves when working on live high-voltage conductors which even in warm conditions, can result in rapid fatigue and reduced performance and have the potential to increase the risk of MSD development [4]. Work in the cold exacerbates these problems [2].

The purpose of the study was therefore to document the challenge of working in the cold wearing the standard five-finger rubber gloves and covers (Standard) and compare them to two equipment options, mitten style gloves (Mitten) or a prototype wool liner (Wool), and two heating options, glove (H-Warmer) or torso (T-Warmer) heating.

\section{Methods}

The independent variables were the 5 glove conditions and the dependent measures were grip force, skin temperature, hand dexterity, completion time of a common task, finger sensitivity, perceived exertion and thermal sensation.

Rubber gloves are manufactured to a regulated safety standard. For the participants recruited, the gloves were available in all sizes enabling participants to wear a properly fitted glove [3]. The glove conditions were: A) Five-finger glove with a regular cotton liner and gauntlet (leather over-glove); B) Mitten gloves with a regular cotton liner and gauntlet and with separate compartments for the thumb, index finger and digits 3-5 with matching covers and manufactured by the same company as the standard glove;

\footnotetext{
* Corresponding author: Richard Wells, Department of Kinesiology, University of Waterloo, 200 University Avenue West, Waterloo, Ontario, Canada, N2L 3G1. Tel: +1 5198884567 ext 33069; Fax: +1 519746 6776; E-mail: wells@uwaterloo.ca.
} 
C) Torso Warmers: A total of four heating pads were secured on the lower back using a torso waist band [4]; D) Glove Warmers: They were secured on the back of the hands over the top of glove liners using tape. Two packs were worn on each hand; E) A woolen under-glove, sourced from an outdoor equipment supplier was trialed.

The study population consisted of 10 male participants from 3 local utility companies; average age 41 yrs (SD +/-11.3), height $1825 \mathrm{~mm}$ (SD+/-59), and weight $96.7 \mathrm{~kg}$ (SD +/-6.6) The participants were all experienced in working with these gloves under winter conditions. Participants were required to be free of upper extremity injury, pain, or discomfort. They were dressed in standard winter clothing mandated by the utility.

The working environment was a controlled temperature walk-in chamber. The chamber controls were set at -20 degrees Celsius and a fan was directed onto the participants. Six thermistors were used to measure skin temperature and were placed on the back of the 3rd and 5th digits, the back of the hand, the back of the forearm, the big toe and the sternum. The participants filled out a rating of perceived effort. The participant's perception of their hand temperature was measured at the completion of each task using a 9 point scale. A Von Frey hair test was administered after 45 mins. They performed simulated utility work for 45 minutes with interspersed test batteries every 15 minutes. These were performed in the test chamber.

It was anticipated that the temperature of the skin over time would mimic that of a first order system and be described by an exponential decrease. All temperature vs time data were therefore transformed with a natural logarithm. The model fit and linearity of the resulting data were checked using linear regression. Very good model fits as evidenced by a high $\mathrm{R}^{2}$ confirmed the appropriateness of the transformation. This also allowed the use of participants who did not complete a condition. The slope of the transformed linear regression was used as the dependent skin temperature variable and one way ANOVA over glove conditions with repeated measures within participants was performed. A Tukey or a Dunnett's post hoc test with condition A, the fivefinger glove, as the control condition followed.

\section{Results}

For the standard five-finger glove, it was found that under conditions of $-20^{\circ} \mathrm{C}$, with air movement simulating wind, and 45 minutes of working on typical tasks, the 5th digit temperature dropped to an average of $14.4{ }^{\circ} \mathrm{C}$ over all participants and conditions. During nine of the 50 trials, one of the participants' skin temperatures -usually the 5th digit- dropped below $10^{\circ} \mathrm{C}$ or the participant did not wish to continue, curtailing the trial before 45 minutes. The mitten style glove or woolen liner in a standard glove reduced the effects of working in the cold compared to the standard five-fingered variety with a thin cotton liner. We found that the mitten style glove showed lesser drops in skin temperature for the 3rd and 5 th digits than the other conditions $(p<0.05)$ (Table 1 and Figure 1). There were neither statistically significant effects nor any apparent trends in skin temperatures or performance for either heating condition.

There was a difference in the initial strength of the five glove conditions, however, there was no statistically significant reduction in strength over the 45 minutes of testing (Table 1). The standard glove showed higher grip forces than all other gloves. The lack of change in maximum strength during the trial might be surprising given the very cold finger temperatures seen, however the power grip strength measured is generated primarily by the extrinsic flexors in the forearm and there was little drop in forearm temperature.

\section{Discussion}

The testing showed that the woolen glove and the mitten glove showed less drop in finger temperature than the standard glove and the heating conditions. The woolen under-glove appears to combine better comfort and interference with performance coupled with less decrease in finger temperature.

Despite the very cold finger temperatures observed, performance degraded surprisingly little, perhaps because of the skilled participants who had learned to compensate for changes in their motor performance. 
Table 1 Effect of the five glove conditions on the dependent measures. ${ }^{1}$ Slope of log transformed data, ${ }^{2}$ Slope in natural units, ${ }^{3}$ Dunnett's post hoc test, ${ }^{4}$ Friedman's chi-square comparing thermal sensation (scale of +4 to -4$)$, ${ }^{5}$ Initial conditions commence at value measured at the start of the day

\begin{tabular}{|c|c|c|c|}
\hline $\begin{array}{l}\text { Dependen } \\
\text { variable }\end{array}$ & & $\mathbf{p}<$ & Post hoc $^{3}$ \\
\hline \multirow{6}{*}{$\begin{array}{l}\text { Skin temp } \\
\text { change } \\
{ }^{\circ} \mathrm{C} / \mathbf{m i n}^{1,5}\end{array}$} & $5^{\text {th }}$ Finger & 0.003 & $\begin{array}{l}\mathrm{A} \text { and } \mathrm{B} \\
\mathrm{B} \text { and } \mathrm{D}\end{array}$ \\
\hline & $3^{\text {rd }}$ Finger & 0.007 & $\begin{array}{l}\mathrm{A} \text { and } \mathrm{B} \\
\mathrm{B} \text { and } \mathrm{D}\end{array}$ \\
\hline & Back of Hand & 0.605 & - \\
\hline & Forearm & 0.436 & - \\
\hline & Chest & 0.575 & - \\
\hline & $1^{\text {st }}$ Hallux & 0.393 & - \\
\hline \multirow[t]{2}{*}{$\begin{array}{l}\text { Max Grip } \\
\text { Force }\end{array}$} & ${ }_{\mathrm{N}}$ Initial Force & $<.001$ & $\begin{array}{l}A \text { and } B \\
A \text { and } C \\
A \text { and } D\end{array}$ \\
\hline & Slope N/min ${ }^{2}$ & 0.191 & None \\
\hline $\begin{array}{l}\text { Semmes } \\
\text { Wein-stein }^{5}\end{array}$ & Change pre-post & 0.523 & - \\
\hline $\begin{array}{l}\text { Hand temp } \\
\text { rating }\end{array}$ & $\begin{array}{l}\text { Change category } \\
/ \mathrm{min}^{2,4}\end{array}$ & $\begin{array}{l}A \text { and B } \\
A \text { and C } \\
A \text { and D } \\
A \text { and } E\end{array}$ & \\
\hline
\end{tabular}

A trade off was seen whereby the participants' hands stayed warmer with the mitten style but with a reduced performance overall. As previously noted, all equipment used by powerline maintainers must be non-flammable or natural fibre. Conditions A, B, and $\mathrm{E}$, fulfilled these conditions. The warmers were trialed in this experiment but would not be permitted when working on live conductors in their present form.

Fig 1. Temperature vs time plot for the $5^{\text {th }}$ digit. Statistical significance testing was performed on the slope of the log transformed data. Differences * indicated $(\mathrm{p}<0.05)$

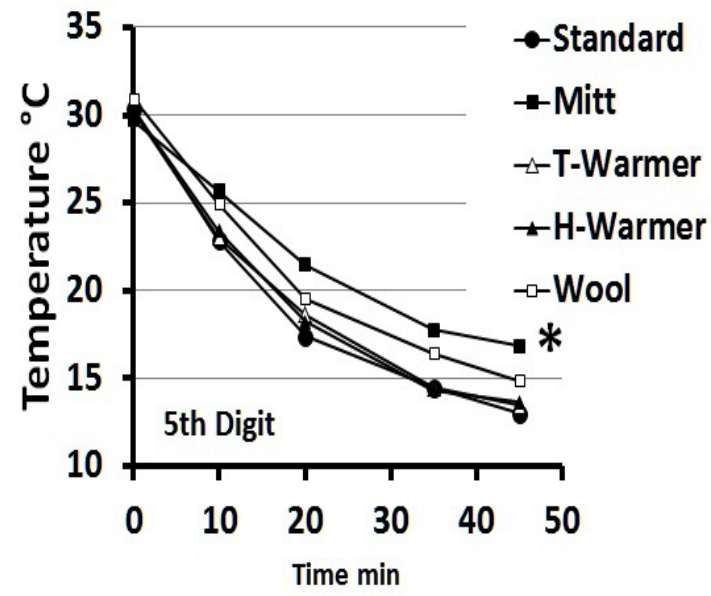

\section{Acknowledgements}

Financial support for this research was provided by a grant from the Workplace Safety and Insurance Board, Ontario.

\section{References}

[1] D. Brajkovic, M. Ducharme, J. Frim, Influence of localized auxiliary heating on hand comfort during cold exposure. Journal of Applied Physiology 85 (1998), 2054-2065.

[2] Q. Geng, F. Chen, I. Holmer, The Effect of protective gloves on manual dexterity in the cold environments. International Journal of Occupational Safety and Ergonomics 3(1-2) (1997), 15-29

[3] R. Wells, S. Hunt, K. Hurley, P. Rosati, Laboratory assessment of the effect of heavy rubber glove thickness and sizing on effort, performance and comfort., International Journal of Industrial Ergonomics 40 (2010),386-391.

[4] K. Willms, R. Wells and H. Carnahan, Determinants of force decrement in gloved power grip, Human Factors 51 (2009), 797-812. 